$\underline{\text { Preprint typeset in JHEP style. - HYPER VERSION }}$

MIT-CTP-2977

IPNO/DR-0010

\title{
Flavour Stability of the Chiral Vacuum and Scalar Meson Dynamics
}

\author{
Bachir Moussallam* \\ Center for Theoretical Physics \\ Massachusetts Institute of Technology \\ Cambridge, MA 02139
}

\begin{abstract}
Previous work relating the flavour variation of the chiral order parameters $F_{\pi},\langle\bar{u} u\rangle$ and $\mathrm{S}$-wave scattering data, based on chiral sum rules and chiral perturbation theory at order $p^{4}$, is extended to include $O\left(p^{6}\right)$ corrections. The finding of a significant decrease of these order parameters, particularly $\langle\bar{u} u\rangle$, with the number of flavours increasing from $N_{F}=2$ to $N_{F}=3$ is confirmed, modulo an assumption on the convergence of the chiral expansion. The connection between scalar resonance physics and the phase structure of the chiral vacuum is also illustrated on the basis of the linear sigma-model. We allow for a very general symmetry breaking sector compatible with softness. The result depends strongly on the input scalar meson masses, in particular, on the presence, or not, of a light sigma.
\end{abstract}

KEYWORDs: chiral lagrangians, sum rules.

\footnotetext{
${ }^{*}$ Permanent address: Groupe de Physique Théorique, IPN Bat100, Université Paris-Sud, 91406 Orsay, France
} 


\section{Contents}

1. Introduction 1

2. $L_{4}, L_{6}$ beyond $O\left(p^{4}\right)$

2.1 Evolution of $F_{\pi}$

2.2 Evolution of $\langle\bar{u} u\rangle$

3. The LEC's in a linear sigma-model 10

3.1 General scalar meson lagrangian 10

3.2 Parameters of the linear sigma-model 13

3.3 Phenomenological applications 16

3.3.1 Light $\sigma$ meson 18

3.3.2 Heavy $\sigma$ meson 19

4. Conclusions 21

A. The correlation function $\Pi_{6}(s)$ to $O\left(p^{6}\right)$

\section{Introduction}

Chiral perturbation theory for $N_{F}=3$ light flavours at next-to-leading order was shown by Gasser and Leutwyler [1] to involve ten independent coupling constants $L_{i}^{r}(\mu)$ (referred to, sometimes, as LEC's). In ref.[1] all ten couplings were determined from low-energy data except two, $L_{4}$ and $L_{6}$. According to the OZI rule or, alternatively, large $N_{c}$ considerations these couplings are expected to be suppressed relative to the other ones [1]. Physically, $L_{4}$ and $L_{6}$ control how observables of the pion, like $M_{\pi}$ or chiral $S U(2)$ order parameters $\left(F_{\pi}\right)_{S U(2)},\langle\bar{u} u\rangle_{S U(2)}$ vary if the mass of the strange quark mass $m_{s}$ varies. If OZI suppression holds these quantities are expected to be essentially insensitive to variations of $m_{s}$ and the value of the chiral order parameters $F_{\pi},\langle\bar{u} u\rangle$ in the $S U(2)$ chiral limit or in the $S U(3)$ chiral limit (i.e. $\left.m_{s}=0\right)$ ought to be very nearly the same. In nature, of course, the values of the light quark masses are fixed but they can be made to vary in unquenched lattice simulations of QCD.

There are reasons to suspect that the couplings $L_{4}$ and $L_{6}$ are, in fact, far from being suppressed. One reason is suggested by two recent unquenched lattice simulations which have investigated the phase structure of QCD-like theories with 
$N_{F}$ equal-mass flavours when the value of $N_{F}$ is varied. It was found in ref. 2] that for $N_{F}>6$ a phase with no confinement and no spontaneous chiral symmetry breaking prevails, at any value of the QCD coupling constant. In ref.[3] a very strong decrease of the chiral order parameters was observed upon varying $N_{F}$ from $N_{F}=2$ to $N_{F}=4$. The values of the coupling constants $L_{i}$ encode informations on the physics of the massive states in QCD [4]. In particular, $L_{4}, L_{6}$ are linked to the scalar resonances. It is notorious that the OZI or large $N_{c}$ rules seem to fail in this sector. In fact, is not clear at present exactly which of the scalar resonances form the lowest lying nonet (see e.g. the reviews in the pdg[5]). In other words, the fact that $L_{4}, L_{6}$ may be unsuppressed, the failure of the OZI rule in the scalar sector are connected and these features may be related in an interesting way to the phase structure of QCD, in particular to the fact that a phase transition could occur for a value of $N_{F}$ not exceedingly larger than $N_{F}=3$. An interpretation of these features in terms of a paramagnetic effect of the quark loops is discussed in ref. [6]

These topics have started to be investigated in a previous paper [7]. The couplings $L_{4}, L_{6}$ were related to the scalar resonances via the scalar form-factors of the pion and the Kaon. These form-factors can be reconstructed directly from experimental data on S-wave scattering, modulo a few plausible hypothesis [8]. The coupling $L_{4}$ is related to the derivative of the strange form-factor of the pion at the origin and, for $L_{6}$, a chiral sum rule can be derived in the terms of the correlator $\Pi_{6}$ of the strange scalar current $\bar{s} s$ and the non-strange current $\bar{u} u+\bar{d} d$. In the present paper, we investigate the $O\left(m_{s}\right)$ corrections to the results of ref. [7], which could be sizable. For this purpose, one needs to use CHPT at order $p^{6}$. Renormalizability of the theory at this order was recently proved[9], and the set of independent chiral lagrangian terms classified[10]. In particular, we will use the expansions of $M_{\pi}$ and $F_{\pi}$ which were derived in refs. 111 and [12] and we have computed the $O\left(p^{6}\right)$ expansion of the correlator $\Pi_{6}$. The result of this effort, at first sight, will turn out to be mitigated: as new coupling constants appear in the formulas, there is a loss in predictivity over the leading order calculation. As discussed in sec.2, conclusions may nevertheless be drawn provided one makes some assumptions on the convergence of the chiral expansion.

In order to gain further insight, we have also investigated a different, more model dependent approach in sec.3. Following the idea of ref. 4] one starts from a lagrangian for the scalar resonances and the LEC's are generated by integrating out the resonances. If we attempt to determine some $O\left(p^{6}\right)$ LEC's in this way, from the most general lagrangian, we find that there are many undetermined resonance parameters which get involved, like three resonance couplings, couplings of one resonance to two scalar sources etc... In sec.3, we discuss the more specific dynamics generated by the linear sigma-model 13]. This model is still attracting interest 14] 15 in connection with the phenomenon of disordered chiral condensate[16] which could be formed in heavy ion collisions. In the symmetry breaking sector, we will consider a lagrangian 
more general than previously done, still compatible with the criterion of softness (restricting ourselves to first order symmetry breaking). The model can accommodate exactly, in principle, a given set of scalar nonet masses and one obtains the $O\left(p^{4}\right)$ and $O\left(p^{6}\right)$ LEC's as definite predictions. The question at this point is which scalar mesons are to be included in the nonet? In particular, does one have to include a light $\sigma$ and a light $\kappa$ meson? It has long been known that an S-matrix pole can be identified in low-energy $\pi \pi$ scattering 18 with a very large imaginary part. A similar structure was argued to exist also in $\pi K$ scattering 19 [20, but it is unclear whether such poles should be interpreted as physical scalar resonances. For our purposes, we will leave this question open and consider two different possibilities for the scalar nonet. As we will see, in the framework of the sigma-model, these will correspond to rather different behaviour of the chiral vacuum.

\section{2. $L_{4}, L_{6}$ beyond $O\left(p^{4}\right)$}

Our estimates of $L_{4}$ and $L_{6}$ are based, essentially, on a method to extract the scalar form-factors of the pion and the kaon using experimental $\pi \pi-K \bar{K}$ scattering data, proposed in ref. [8]. The form-factors get determined up to a normalization factor, for which one uses CHPT. In order to determine $L_{4}$ one equals the experimental determination of $G_{\pi}^{\prime}(0)$, the derivative of the strange scalar form-factor of the pion and the chiral expansion of this quantity. In ref.[7] this matching was performed at leading order in the chiral expansion, i.e. $G_{\pi}^{\prime}(0)$ was expanded up to $O\left(p^{4}\right)$ and for the normalization condition we used CHPT at order $p^{2}$. In a similar way, for $L_{6}$ we use the scalar form factors to express the spectral function of the correlator $\Pi_{6}(s)$ (see (2.13) below) and $L_{6}$ is obtained by matching $\Pi_{6}(0)$ evaluated from experimental data and its chiral expansion. Below, we discuss the chiral corrections to these results, which involve two parts a) one must use the chiral expansions of $G_{\pi}^{\prime}(0)$ and $\Pi_{6}(0)$ up to $O\left(p^{6}\right)$ and b) we must use CHPT at $O\left(p^{4}\right)$ instead of $O\left(p^{2}\right)$ in the normalization conditions.

Let us designate by $F_{\pi}(s)$ and $G_{\pi}(s)$ the non-strange and strange scalar form factors of the pion, and by $F_{K}(s)$ and $G_{K}(s)$ the analogous ones for the Kaon. We will use the following normalizations,

$$
\begin{array}{rlrl}
F_{\pi} & =\frac{1}{B_{0}} \sqrt{\frac{3}{2}}\left\langle 0|\bar{u} u+\bar{d} d| \pi^{0} \pi^{0}\right\rangle & G_{\pi} & =\frac{1}{B_{0}} \sqrt{\frac{3}{2}}\left\langle 0|\bar{s} s| \pi^{0} \pi^{0}\right\rangle \\
F_{K}=\frac{1}{B_{0}} \sqrt{2}\left\langle 0|\bar{u} u+\bar{d} d| K^{+} K^{-}\right\rangle & G_{K}=\frac{1}{B_{0}} \sqrt{2}\left\langle 0|\bar{s} s| K^{+} K^{-}\right\rangle .
\end{array}
$$

The method of ref. [8] consists in solving numerically a set of Muskhelishvili-Omnès coupled-channel equations using experimentally determined $\pi \pi-K \bar{K}$ S-wave scattering T-matrix elements as input(in practice we made use of the parametrisations of ref. 21] and ref. 22]). Strictly speaking, these equations hold under the assumption of 
exact two-channel unitarity up to $s=\infty$ while in practice, two-channel unitarity is a good approximation up to the $\eta \eta$ threshold. The resulting form factors are expected to be reliable in a finite energy range, which we will assume to extend up to $1 \mathrm{GeV}$. One also chooses appropriate boundary conditions for the T-matrix at $s=\infty$ which insure existence of a solution with a minimal number of free parameters: a solution vector $u_{1}(s), u_{2}(s)$ will be determined in the entire energy range once the values at one point, say at $s=0$, are given. Extension to more channels and the stability of this scheme were discussed in ref. [7]. In practice, one first constructs numerically two independent solutions of the equation set: $u_{i}(s)$ and $v_{i}(s), i=1,2$, normalized at the origin such that $u_{1}(0)=1, u_{2}(0)=0$ and $v_{1}(0)=0, v_{2}(0)=1$. The form factors are then given as

$$
\left(\begin{array}{l}
F_{\pi}(s) \\
F_{K}(s)
\end{array}\right)=F_{\pi}(0)\left(\begin{array}{l}
u_{1}(s) \\
u_{2}(s)
\end{array}\right)+F_{K}(0)\left(\begin{array}{l}
v_{1}(s) \\
v_{2}(s)
\end{array}\right)
$$

and similarly for the strange form factors. In refs. [8] [0] the normalization at the origin was taken from CHPT at $O\left(p^{2}\right)$. Here, we wish to investigate the chiral corrections to these results, so we need to go to the next chiral order.

\subsection{Evolution of $F_{\pi}$}

In the $S U(2)$ chiral limit, the strange form factor of the pion vanishes at the origin $G_{\pi}(0)=0$, the LEC $L_{4}$ is related to the first derivative at the origin of $G_{\pi}$. It is convenient to consider the proportional quantity

$$
d_{F}^{e x p}=\sqrt{\frac{2}{3}} m_{s} B_{0} G_{\pi}^{\prime}(0) \equiv \sqrt{\frac{2}{3}} m_{s} B_{0} G_{K}(0) v_{1}^{\prime}(0) .
$$

Here, $v_{1}^{\prime}(0)$ can be determined from experiment by the procedure outlined above, and one finds,

$$
v_{1}^{\prime}(0) \simeq 0.27 \mathrm{GeV}^{-2},
$$

using the T-matrix parametrisation of ref.[22] (using that of ref. 21] one would find $\left.v_{1}^{\prime}(0) \simeq 0.31 \mathrm{GeV}^{-2}\right)$. We next need to express $m_{s} B_{0}$ and $G_{K}(0)$ in eq.(2.3). At leading chiral order, one has $m_{s} B_{0}=m_{K}^{2}-m_{\pi}^{2} / 2$ and $G_{K}(0)=\sqrt{2}$. Including $O\left(m_{s}\right)$ corrections to this result, one obtains $d_{F}^{\exp }$ in the form,

$$
d_{F}^{e x p}=\frac{2}{\sqrt{3}}\left[m_{K}^{2}-m_{\pi}^{2} / 2+\frac{m_{K}^{4}}{F_{\pi}^{2}}\left(-8 S_{8}-16 S_{6}+\frac{1}{36 \pi^{2}}\left(L_{\eta}+1\right)\right)\right] v_{1}^{\prime}(0),
$$

with

$$
S_{8}=-2 L_{8}^{r}(\mu)+L_{5}^{r}(\mu), S_{6}=-2 L_{6}^{r}(\mu)+L_{4}^{r}(\mu), L_{\eta}=\log \frac{m_{\eta}^{2}}{\mu^{2}} .
$$

Corrections of order $m_{\pi}^{2} m_{K}^{2}$ or $m_{\pi}^{4}$ have been neglected here and CHPT at $O\left(p^{4}\right)$ has been used to obtain $G_{K}(0)$ and to express $m_{s} B_{0}$ in terms of the physical Kaon 
mass. We observe that low-energy constants appear now but it its consistent to use their values obtained at $O\left(p^{4}\right)$, i.e. for $L_{5}, L_{8}$ the values given in ref.[1] and for $L_{4}$, $L_{6}$ those obtained in ref. [7]. We can then match $d_{F}^{\exp }$ with its expression as a chiral expansion. The latter is obtained from the relation 8

$$
d_{F}=\frac{m_{s}}{F_{\pi}} \frac{d F_{\pi}}{d m_{s}}
$$

which holds in the chiral $S U(2)$ limit, $m_{u}=m_{d}=0$, and one can use the chiral expansion of $F_{\pi}$ which has been determined up to $O\left(p^{6}\right)$ [1] [12]. One obtains

$$
d_{F}=\frac{m_{s} B_{0}}{F_{0}^{2}} d^{(4)}+\frac{m_{s}^{2} B_{0}^{2}}{F_{0}^{4}} d^{(6)}+O\left(m_{s}^{3}\right),
$$

with

$$
d^{(4)}=8 L_{4}^{r}-\frac{1}{32 \pi^{2}}\left(L_{K}+1\right), \quad L_{K}=\log \frac{m_{s} B_{0}}{\mu^{2}}
$$

and

$$
\begin{aligned}
d^{(6)}= & 64 C_{16}^{r}-128\left(L_{4}^{r}\right)^{2}-\frac{17}{3072 \pi^{4}} L_{K}^{2}-\frac{5}{1152 \pi^{4}} L_{K} L_{\eta} \\
& +\frac{L_{K}}{\pi^{2}}\left(\frac{73}{9216 \pi^{2}}+4 L_{1}^{r}+L_{2}^{r}+\frac{5}{4} L_{3}^{r}+\frac{1}{2} L_{5}^{r}-2 L_{6}^{r}-L_{8}^{r}\right) \\
& +\frac{L_{\eta}}{\pi^{2}}\left(-\frac{1}{768 \pi^{2}}+\frac{16}{9} L_{1}^{r}+\frac{4}{9} L_{2}^{r}+\frac{4}{9} L_{3}^{r}-\frac{8}{9} L_{4}^{r}\right) \\
& +\frac{1}{\pi^{2}}\left(\frac{26}{9} L_{1}^{r}+\frac{35}{54} L_{3}^{r}+\frac{5}{9} L_{4}^{r}+\frac{3}{4} L_{5}^{r}-3 L_{6}^{r}-\frac{3}{2} L_{8}^{r}-\frac{0.0022}{\pi^{2}}\right) .
\end{aligned}
$$

In this formula, the finite contribution from the so-called sunset diagrams has been evaluated numerically. Equating (2.3) and (2.8) at the leading, linear order in $m_{s}$, gives the $O\left(p^{4}\right)$ determination of $L_{4}$. From the numerical results of refs. [8] [7] one obtains

$$
L_{4}^{r}\left(m_{\eta}\right) \simeq 0.610^{-3} \quad\left[\text { order } p^{4}\right]
$$

If we include the corrections quadratic in $m_{s}$ now, a new $O\left(p^{6}\right)$ coupling constant appears, labelled $C_{16}$ in ref. [10], so strictly speaking, we have one equation and two unknowns. A similar situation will prevail in the determination of $L_{6}$ to be discussed below. We expect, however, the chiral expansion to be meaningful and the $O\left(p^{6}\right)$ part of the expansion to be smaller than the $O\left(p^{4}\right)$ one. If we assume a given ratio for these two parts we can determine $L_{4}$ and $C_{16}$ separately. Once these two couplings are known we can, furthermore, using the CHPT expressions of ref.[11] [12], determine how $F_{\pi}$ varies in going from an $S U(2)$ chiral limit to an $S U(3)$ one. Some results are collected in table 1 , where we have varied the $O\left(p^{6}\right)$ over $O\left(p^{4}\right)$ ratio between $10 \%$ and $100 \%$.

This exercise seems to indicate that the determination of $L_{4}$ is rather stable and rather close to its determination at leading order, and provides an estimate for the 


\begin{tabular}{|c|c|c|c|}
\hline$O\left(p^{6}\right) / O\left(p^{4}\right)$ & $10^{3} L_{4}$ & $10^{5} C_{16}$ & $F / F_{0}-1$ \\
\hline 0.10 & $0.58[0.31]$ & $0.36[0.24]$ & $0.18+0.005$ \\
0.30 & $0.53[0.26]$ & $0.39[0.26]$ & $0.17+0.011$ \\
0.50 & $0.49[0.22]$ & $0.41[0.28]$ & $0.16+0.016$ \\
1 & $0.42[0.15]$ & $0.44[0.32]$ & $0.14+0.024$ \\
\hline
\end{tabular}

Table 1: Assuming a given ratio of the $O\left(p^{6}\right)$ to the $O\left(p^{4}\right)$ contributions in the chiral expansion of $d_{F}$, the values of the LEC's $L_{4}^{r}(\mu)$ and $C_{16}^{r}(\mu)$ are computed for $\mu=m_{\eta}$ (in brackets, $\mu=m_{\rho}$ ). We also display the successive contributions to the ratio of $F \equiv$ $\left(F_{\pi}\right)_{S U(2)}$ over $F_{0} \equiv\left(F_{\pi}\right)_{S U(3)}$

size of the $O\left(p^{6}\right)$ coupling constant $C_{16}^{r}$. A priori, however, one cannot completely exclude a different solution, with $L_{4}\left(m_{\eta}\right)=0$, for instance. In this case, all the OZI violation would be concentrated in the $O\left(p^{6}\right)$ parameter, which seems somewhat unplausible. Taking into account the experimental uncertainties in the T-matrix, the uncertainty from the energy region above $1 \mathrm{GeV}$ (which were estimated in ref. [7]) and the discussion above of the $O\left(m_{s}\right)$ corrections, we find that the error on $L_{4}$ should be of the order of $30 \%$, i.e.,

$$
L_{4}\left(m_{\eta}\right)=(0.6 \pm 0.2) 10^{-3}
$$

\subsection{Evolution of $\langle\bar{u} u\rangle$}

Let us now discuss the coupling constant $L_{6}$. Using the same scalar form factors, it was remarked in ref.[7] that one can derive an estimate of $L_{6}$. The idea is to consider the two-point correlation function,

$$
\Pi_{6}\left(p^{2}\right)=\frac{i}{B_{0}^{2}} \int d^{4} x \mathrm{e}^{i p x}\langle 0|T(\bar{u} u(x)+\bar{d} d(x)) \bar{s} s(0)| 0\rangle_{\text {conn } .} .
$$

In an energy domain extending roughly up to the $\eta \eta$ threshold, the spectral function is given in terms of the scalar form factors of the pion and the Kaon,

$$
\begin{aligned}
\operatorname{Im}_{6}(s)= & \sqrt{\frac{s-4 m_{\pi}^{2}}{s}} F_{\pi}(s) G_{\pi}^{*}(s) \theta\left(s-4 m_{\pi}^{2}\right) \\
& +\sqrt{\frac{s-4 m_{K}^{2}}{s}} F_{K}(s) G_{K}^{*}(s) \theta\left(s-4 m_{K}^{2}\right) .
\end{aligned}
$$

Essentially, a usual assumption is made there, that the $4 \pi$ or $6 \pi$ contributions are negligibly small in this energy region (see, e.g. 21]). In the $S U(2)$ chiral limit, $m_{u}=$ $m_{d}=0$, a super-convergence relation holds,

$$
\int_{0}^{\infty} \operatorname{Im} \Pi_{6}(s) d s=0
$$


Similar chiral sum rules are known to be saturated to a fairly good approximation in terms of a few low-lying resonances (see, e.g. 23]). In the energy region below $1 \mathrm{GeV}$, inserting the form factors constructed as discussed above, the contribution to the integral is found to be positive, and dominated by the $f_{0}(980)$ resonance. A plausible assumption, then, is that the higher energy contribution to the sum rule (2.15) will be saturated, at least approximately, by the next prominent scalar resonance, the $f_{0}(1500)$, which we expect to make a negative contribution. We observe that the signs of the contributions of the $f_{0}(980)$ and the $f_{0}(1500)$ conforms with the recent assignments of Minkowski and Ochs 24 as (essentially) $S U_{F}(3)$ singlet and octet respectively. Finally, one can calculate the value of the correlation function at zero energy, $\Pi_{6}(0)$, making use of the scalar form factors below $1 \mathrm{GeV}$, and the superconvergence relation (2.15) as,

$$
\Pi_{6}(0) \simeq \frac{1}{\pi}\left(\int_{0}^{1} \frac{\operatorname{Im} \Pi_{6}(s)}{s} d s-\frac{1}{s_{0}} \int_{0}^{1} \operatorname{Im} \Pi_{6}(s) d s\right)
$$

with $s_{0} \simeq 1.5^{2} \mathrm{GeV}^{2}$. This gives us an "experimental" value of $\Pi_{6}(0)$, the difference with ref. [7] is that we now wish to take into account chiral corrections in the normalization of the form factors. Below the $K \bar{K}$ threshold, we can express the spectral function in terms of the basis solutions to the Muskhelishvili-Omnès equations $u_{i}(s)$, $v_{i}(s)$ as,

$$
\begin{aligned}
\operatorname{Im} \prod_{6}(s)=\sqrt{\frac{s-4 m_{\pi}^{2}}{s}}[ & F_{\pi}(0) G_{K}(0) u_{1}(s) v_{1}^{*}(s) \\
& \left.+F_{K}(0) G_{K}(0) v_{1}(s) v_{1}^{*}(s)\right] .
\end{aligned}
$$

Including $O\left(m_{s}\right)$ chiral corrections in the normalizing factors, we now have

$$
\begin{aligned}
& F_{\pi}(0) G_{K}(0)=2 \sqrt{3}\left(1+\frac{m_{K}^{2}}{F_{\pi}^{2}}\left[-16 S_{8}-48 S_{6}+\frac{1}{24 \pi^{2}} L_{\eta}+\frac{1}{36 \pi^{2}}\right]\right) \\
& F_{K}(0) G_{K}(0)=2\left(1+\frac{m_{K}^{2}}{F_{\pi}^{2}}\left[-32 S_{8}-80 S_{6}+\frac{7}{72 \pi^{2}} L_{\eta}+\frac{1}{24 \pi^{2}}\right]\right)
\end{aligned}
$$

where $S_{8}=-2 L_{8}^{r}+L_{5}^{r}, S_{6}=-2 L_{6}^{r}+L_{4}^{r}$ and we may use in this part the values of the LEC's as determined at $O\left(p^{4}\right)$.

We then use the experimental result for $\Pi_{6}(0)$ in conjunction with its chiral expansion. The computation of $\Pi_{6}(s)$ to $O\left(p^{6}\right)$ is exposed in the appendix. Concerning $\Pi_{6}(0)$, the chiral expansion goes as follows

$$
\Pi_{6}(0)=\Pi^{(4)}+\frac{m_{s} B_{0}}{F_{0}^{2}} \Pi^{(6)},
$$

with

$$
\Pi^{(4)}=64 L_{6}^{r}-\frac{1}{16 \pi^{2}}\left(\frac{22}{9}\left(L_{K}+1\right)+\frac{4}{9} L_{43}\right)
$$


and

$$
L_{K}=\log \frac{m_{s} B_{0}}{\mu^{2}}, \quad L_{43}=\log \frac{4}{3} .
$$

This expression generates the $O\left(p^{4}\right)$ determination[[]] of $L_{6}$,

$$
L_{6}^{r}\left(m_{\eta}\right) \simeq 0.510^{-3} \quad\left[\text { order } p^{4}\right] .
$$

The expression of the $O\left(p^{6}\right)$ part in (2.19) is,

$$
\begin{aligned}
\Pi^{(6)}= & 256\left(C_{20}^{r}+3 C_{21}^{r}\right)-\frac{1}{72 \pi^{4}} L_{K}^{2}-\frac{1}{96 \pi^{4}} L_{K} L_{43}+\frac{1}{288 \pi^{4}} L_{43}^{2} \\
& +\frac{L_{K}}{\pi^{2}}\left(\frac{64}{3} S_{6}+\frac{70}{9} S_{8}+\frac{16}{9} S_{7}-\frac{55}{2592 \pi^{2}}\right) \\
& +\frac{L_{43}}{\pi^{2}}\left(\frac{16}{3} S_{6}+\frac{16}{9} S_{8}+\frac{16}{9} S_{7}-\frac{47}{5184 \pi^{2}}\right) \\
& +\frac{1}{\pi^{2}}\left(\frac{140}{9} S_{6}+\frac{175}{27} S_{8}+\frac{8}{27} S_{7}-\frac{19}{5184 \pi^{2}}\right) .
\end{aligned}
$$

with

$$
S_{7}=3 L_{7}+L_{8}^{r}
$$

In these formulas, we have set $m_{u}=m_{d}=0$. These expressions exhibit the explicit $m_{s}$ dependence (which will prove usefull below as we plan to integrate over $m_{s}$ ). For numerical application, we may use $m_{s} B_{0}=m_{K}^{2}-m_{\pi}^{2} / 2$ in $\Pi^{(6)}$ while in $\Pi^{(4)}$ we have to use the $O\left(p^{4}\right)$ expansions of $m_{K}^{2}$ and $m_{\pi}^{2}[\mathbb{1}$. Equating the chiral expansion of $\Pi_{6}(0)$ with its sum rule evaluation gives us a linear relation involving the $O\left(p^{4}\right)$ LEC $L_{6}$ and the $O\left(p^{6}\right)$ combination $C_{20}+3 C_{21}$. As before, we must make a hypothesis concerning the convergence of the chiral expansion if we want to separately estimate these two contributions.

Another interesting information contained in the correlation function $\Pi_{6}$ concerns the variation of the quark condensate in the chiral $S U(2)$ limit as a function of the strange quark mass:

$$
\frac{d\langle\bar{u} u\rangle}{d m_{s}}=-\frac{B_{0}^{2}}{2} \Pi_{6}(0) .
$$

Using the chiral expansion of $\Pi_{6}(0)$ we can integrate this equation in the variable $m_{s}$ from its physical value down to $m_{s}=0$. In this way we obtain an estimate for how the condensate varies from a chiral $S U(2)$ limit to a chiral $S U(3)$ limit. This variation is obtained as an expansion in powers of the physical strange quark mass (or, alternatively, in powers of the Kaon mass),

$$
\langle\bar{u} u\rangle_{S U(2)}=\langle\bar{u} u\rangle_{S U(3)}\left(1+\frac{m_{s} B_{0}}{F_{0}^{2}} R^{(4)}+\frac{m_{s}^{2} B_{0}^{2}}{F_{0}^{4}} R^{(6)}\right),
$$

with

$$
R^{(4)}=32 L_{6}^{r}-\frac{11}{144 \pi^{2}} L_{K}-\frac{1}{72 \pi^{2}} L_{43}
$$


and

$$
\begin{aligned}
R^{(6)}= & 64\left(C_{20}^{r}+3 C_{21}^{r}\right)-\frac{1}{288 \pi^{4}} L_{K}^{2}-\frac{1}{384 \pi^{4}} L_{K} L_{43}+\frac{1}{1152 \pi^{4}} L_{43}^{2} \\
& +\frac{L_{K}}{\pi^{2}}\left(\frac{16}{3} S_{6}+\frac{4}{9} S_{7}+\frac{35}{18} S_{8}-\frac{19}{10368 \pi^{2}}\right) \\
& +\frac{L_{43}}{\pi^{2}}\left(\frac{4}{3} S_{6}+\frac{4}{9} S_{7}+\frac{4}{9} S_{8}-\frac{5}{5184 \pi^{2}}\right) \\
& +\frac{1}{\pi^{2}}\left(\frac{11}{9} S_{6}-\frac{4}{27} S_{7}+\frac{35}{54} S_{8}\right) .
\end{aligned}
$$

Using the relation

$$
-2\langle\bar{u} u\rangle_{S U(2)}=\frac{d}{d m}\left(m_{\pi}^{2} F_{\pi}^{2}\right)_{m=0}, \quad m=\frac{1}{2}\left(m_{u}+m_{d}\right),
$$

one sees that the expansion of the condensate can be rederived from the expansion of the product $m_{\pi}^{2} F_{\pi}^{2}$ in powers of $m_{s}$. We have verified, using the $O\left(p^{6}\right)$ expansion [11] [12] of $m_{\pi}, F_{\pi}$ that the formulas (2.26), (2.27), (2.28) are exactly reproduced.

Let us now discuss some numerical results. At first, it is instructive to compare the value obtained for $\Pi_{6}(0)$ using the normalization of the form factors at $O\left(p^{2}\right)$ and the normalization at $O\left(p^{4}\right)$,

$$
\Pi_{6}(0) \simeq 0.022\left[O\left(p^{2}\right) \text { norm. }\right], \quad \Pi_{6}(0) \simeq 0.043\left[O\left(p^{4}\right) \text { norm. }\right] .
$$

We see that including $O\left(m_{s}\right)$ corrections in the normalizations brings a rather substantial change in the result by approximately a factor of two. This can be traced to the large numbers appearing in front of the combination $S_{6}=-2 L_{6}+L_{4}$ in the normalization factors (2.18). Evidently, the difference $-2 L_{6}+L_{4}$ is very sensitive to a small variation of $L_{6}$ or $L_{4}$. Taking $L_{6}$ slightly smaller than the central value (2.22) would decrease significantly the modification in $\Pi_{6}(0)$ without essentially altering the other results. Upon considering the chiral expansion of $\Pi_{6}(0)$ now, this result suggests that the $O\left(p^{6}\right)$ contribution could be substantial and it is likely to be positive. We have collected some results in table 2 assuming that the ratio of the $O\left(p^{6}\right)$ to the $O\left(p^{4}\right)$ contributions is positive and ranges from $10 \%$ to $100 \%$. In this range, we find that the rate of convergence for the ratio of quark condensates seems reasonable.

Both $F_{\pi}$ and $\langle\bar{u} u\rangle$ show a tendency towards chiral restoration in going from chiral $S U(2)$ to chiral $S U(3)$. This tendency seems much stronger for $\langle\bar{u} u\rangle$. This is in agreement with the arguments of ref.[6] based on the spectrum of the Dirac operator. One must however bear in mind that its dimensionality is different from $F_{\pi}$ : if we had considered $\langle\bar{u} u\rangle^{1 / 3}$, we would have found a smaller variation. This behaviour could perhaps suggest the possibility, at larger $N_{F}$, of a phase with vanishing quark condensate, yet with chiral symmetry still spontaneously broken 25. Kogan et al. 26. have discussed how this could result from a discrete $Z_{2}$ axial subgroup remaining 


\begin{tabular}{|c|c|c|c|}
\hline$O\left(p^{6}\right) / O\left(p^{4}\right)$ & $10^{3} L_{6}$ & $10^{5}\left(C_{20}+3 C_{21}\right)$ & $\langle\bar{u} u\rangle_{S U(2)} /\langle\bar{u} u\rangle_{S U(3)}-1$ \\
\hline 0.10 & $0.73[0.56]$ & $0.29[-0.02]$ & $0.85+0.14$ \\
0.30 & $0.64[0.47]$ & $0.37[0.06]$ & $0.76+0.18$ \\
0.50 & $0.57[0.41]$ & $0.43[0.12]$ & $0.69+0.21$ \\
1 & $0.46[0.30]$ & $0.52[0.21]$ & $0.58+0.26$ \\
\hline
\end{tabular}

Table 2: Assuming a given ratio of the $O\left(p^{6}\right)$ to the $O\left(p^{4}\right)$ contributions in the chiral expansion of $\Pi_{6}(0)$ the values of the LEC's $L_{6}^{r}\left(m_{\eta}\right)$ and the combination $C_{20}^{r}(\mu)+3 C_{21}^{r}(\mu)$ are computed for $\mu=m_{\eta}$ (in brackets, $\mu=m_{\rho}$ ). We also display the successive terms in the expansion of $\langle\bar{u} u\rangle_{S U(2)} /\langle\bar{u} u\rangle_{S U(3)}-1$.

unbroken, but argue against this possibility in QCD. Concerning the error in this evaluation of $L_{6}$, finally, it is expected to be somewhat larger than the error on $L_{4}$ because of a greater sensitivity to the energy region above $1 \mathrm{GeV}$. The discussion above also suggests that one should have $L_{6}\left(m_{\eta}\right)<L_{4}\left(m_{\eta}\right)$ otherwise the $O\left(m_{s}\right)$ corrections could become out of control. Keeping this mind, we find that $L_{6}$ should lie in the following range,

$$
L_{6}\left(m_{\eta}\right)=(0.5 \pm 0.3) 10^{-3}
$$

\section{The LEC's in a linear sigma-model}

\subsection{General scalar meson lagrangian}

Here, we adopt a simple resonance saturation point of view for estimating the lowenergy coupling constants, which was discussed in detail in ref. [4]. It consists in making a tree level calculation starting from a lagrangian for the resonances. We will consider the scalar resonances here. It is most convenient to start from a representation of the resonances in which they transform under a non-linear representation of the chiral group[27]. A detailed discussion can be found in ref. [4], we adopt essentially the same notations here. If one is interested in the $O\left(p^{4}\right)$ LEC's then one needs consider only those terms which are quadratic in the resonance fields,

$$
\mathcal{L}_{S S}=\frac{1}{2} \partial_{\mu} S_{0} \partial^{\mu} S_{0}+\frac{1}{2}\left\langle\nabla_{\mu} S \nabla^{\mu} S\right\rangle-\frac{1}{2} M_{0}^{2} S_{0}^{2}-\frac{1}{2} M_{8}^{2}\left\langle S^{2}\right\rangle,
$$

or containing one resonance field and one scalar source, or one scalar field and two chiral fields $u_{\mu}$, i.e.

$$
\mathcal{L}_{S \chi}=c_{d}\left\langle S u_{\mu} u^{\mu}\right\rangle+\tilde{c}_{d} S_{0}\left\langle u_{\mu} u_{\mu}\right\rangle+c_{m}\left\langle S \chi_{+}\right\rangle+\tilde{c}_{m} S_{0}\left\langle\chi_{+}\right\rangle,
$$

where $S_{0}$ is the singlet scalar, with chiral limit mass $M_{0}, S$ is a traceless matrix encoding the scalar octet, which has a common mass $M_{8}$ in the chiral limit. We 
use the same conventions otherwise as ref. 4 . This lagrangian yields the resonance saturation estimates for the following low-energy constants,

$$
\begin{array}{ll}
L_{4}=-\frac{c_{d} c_{m}}{3 M_{8}^{2}}+\frac{\tilde{c}_{d} \tilde{c}_{m}}{M_{0}^{2}}, & L_{5}=\frac{c_{d} c_{m}}{M_{8}^{2}}, \\
L_{6}=-\frac{c_{m}^{2}}{6 M_{8}^{2}}+\frac{\tilde{c}_{m}^{2}}{2 M_{0}^{2}}, & L_{8}=\frac{c_{m}^{2}}{2 M_{8}^{2}} .
\end{array}
$$

In addition, the scalar resonances make contributions to $L_{1}, L_{3}$,

$$
L_{1}=-\frac{c_{d}^{2}}{6 M_{8}^{2}}+\frac{\tilde{c}_{d}^{2}}{2 M_{0}^{2}}, \quad L_{3}=\frac{c_{d}^{2}}{2 M_{8}^{2}},
$$

which receive other important contributions from the vector and axial-vector resonance sector. These tree-level estimates yield coupling constants which are scale independent $^{1}$ : one usually assumes that they should represent meaningful estimates of the $L_{i}^{r}(\mu)$ for values of $\mu$ for which chiral logarithms are numerically small, that is of the order of $\mu=0.5$ to $\mu=1 \mathrm{GeV}$. In ref. 四 the OZI rule was assumed to apply, implying $L_{4}=L_{6}=0$. The formulas (3.3) provide one relation between the values of $L_{5}, L_{8}$ and the experimental value of the decay width $a_{0} \rightarrow \pi \eta$. Values of the couplings $c_{d}$ and $c_{m}$ were extracted,

$$
\left|c_{d}\right| \simeq 32 \mathrm{MeV} \quad\left|c_{m}\right| \simeq 42 \mathrm{MeV}
$$

Below, we will investigate the more detailed predictions that one can make if one assumes the validity of a renormalizable, sigma-model type interaction lagrangian. Before we discuss this, let us consider the generalisation of the considerations above to LEC's of chiral order $O\left(p^{6}\right)$. We will illustrate the complications which arise at this order by considering only the lagrangian terms which are cubic in the scalar source $\chi_{+}$. These terms involve the three coupling constants 10$] C_{19}, C_{20}, C_{21}$,

$$
\mathcal{L}^{(6)}=\ldots+C_{19}\left\langle\chi_{+}^{3}\right\rangle+C_{20}\left\langle\chi_{+}^{2}\right\rangle\left\langle\chi_{+}\right\rangle+C_{21}\left\langle\chi_{+}\right\rangle^{3}+\ldots
$$

Firstly, one needs to consider interaction terms which are cubic in the resonance fields,

$$
\mathcal{L}_{S S S}=a S_{0}^{3}+b S_{0}\left\langle S^{2}\right\rangle+c\left\langle S^{3}\right\rangle,
$$

then terms which are quadratic in the resonance fields and linear in the scalar source,

$$
\begin{gathered}
\mathcal{L}_{S S \chi}=\stackrel{\circ}{e_{m}}\left\langle S^{2}\right\rangle\left\langle\chi_{+}\right\rangle+\stackrel{\circ}{f}_{m} S_{0}\left\langle S \chi_{+}\right\rangle \\
+\stackrel{\circ}{g}_{m}\left\langle S^{2} \chi_{+}\right\rangle+\stackrel{\circ}{h}_{m} S_{0}^{2}\left\langle\chi_{+}\right\rangle,
\end{gathered}
$$

\footnotetext{
${ }^{1}$ If one starts from the renormalizable sigma-model it is possible, in principle, to compute the one-loop effective action and then generate the $O\left(p^{4}\right)$ LEC's with the correct scale dependence. This was done for the $\mathrm{SU}(2)$ sigma-model in ref. 28
} 
and, finally, terms linear in the scalar field and quadratic in the scalar source,

$$
\begin{aligned}
& \mathcal{L}_{S \chi \chi}=a^{\prime} S_{0}\left\langle\chi_{+}\right\rangle^{2}+b^{\prime} S_{0}\left\langle\chi_{+}^{2}\right\rangle+c^{\prime}\left\langle S \chi_{+}\right\rangle\left\langle\chi_{+}\right\rangle \\
& \quad+d^{\prime}\left\langle S \chi_{+}^{2}\right\rangle .
\end{aligned}
$$

This already brings in a large number of couplings and it would not be possible to make definite predictions without additional assumptions. The terms in eqs.(3.7), (3.8) and (3.9) generate chiral $O\left(p^{6}\right)$ coupling constants as well as chiral symmetry breaking for the scalar meson masses. It is convenient, both for generating the $O\left(p^{4}\right)$ and $O\left(p^{6}\right)$ LEC's and for expressing the scalar meson masses to make the following field redefinitions,

$$
\begin{aligned}
& S_{0} \rightarrow S_{0}+\frac{a}{M_{0}^{2}} S_{0}^{2}+\frac{b}{M_{0}^{2}}\left\langle S^{2}\right\rangle+\frac{\tilde{c}_{m}}{M_{0}^{2}}\left\langle\chi_{+}\right\rangle \\
& S \rightarrow S+\frac{c}{M_{8}^{2}}\left(S^{2}-\frac{1}{3}\left\langle S^{2}\right\rangle I_{d}\right)+\frac{c_{m}}{M_{8}^{2}}\left(\chi_{+}-\frac{1}{3}\left\langle\chi_{+}\right\rangle I_{d}\right)
\end{aligned}
$$

The redefined fields $S_{0}$ and $S$ have, by construction, no trilinear couplings and no minimal one-resonance to one-source coupling. The couplings of two resonances to one scalar source, eq.(3.8), get redefined to

$$
\begin{aligned}
& g_{m}={\stackrel{\circ}{g_{m}}}+\frac{3 c_{m} c}{M_{8}^{2}}, \quad h_{m}=\stackrel{\circ}{h}_{m}+\frac{3 \tilde{c}_{m} a}{M_{0}^{2}}, \\
& e_{m}=\stackrel{\circ}{e}_{m}+\frac{\tilde{c}_{m} b}{M_{0}^{2}}-\frac{c_{m} c}{M_{8}^{2}}, \quad f_{m}=\stackrel{\circ}{f}_{m}+\frac{2 c_{m} b}{M_{8}^{2}} .
\end{aligned}
$$

The scalar meson masses, to linear order in the quark masses, can be straightforwardly expressed in terms of these redefined parameters. The masses of the isospin $I=1$ and $I=1 / 2$ mesons read,

$$
\begin{aligned}
& M_{a_{0}}^{2}=M_{8}^{2}-4 e_{m}\left(2 m_{K}^{2}+m_{\pi}^{2}\right)-4 g_{m} m_{\pi}^{2} \\
& M_{\kappa_{0}}^{2}=M_{a_{0}}^{2}-4 g_{m}\left(m_{K}^{2}-m_{\pi}^{2}\right),
\end{aligned}
$$

while the isospin zero meson masses diagonalise the singlet-octet mass matrix which gets parametrised as follows

$$
\begin{aligned}
& \mathcal{M}_{11}=M_{0}^{2}-4 h_{m}\left(2 m_{K}^{2}+m_{\pi}^{2}\right) \\
& \mathcal{M}_{12}=4 f_{m} \sqrt{\frac{2}{3}}\left(m_{K}^{2}-m_{\pi}^{2}\right) \\
& \mathcal{M}_{22}=M_{a_{0}}^{2}-\frac{16}{3} g_{m}\left(m_{K}^{2}-m_{\pi}^{2}\right) .
\end{aligned}
$$

In deriving these expressions for the masses, we have ignored possible terms in the scalar lagrangian of the form $\partial_{\mu} S_{0} \partial^{\mu} S_{0}\left\langle\chi_{+}\right\rangle,\left\langle\nabla_{\mu} S \nabla^{\mu} S \chi_{+}\right\rangle$, anticipating on the fact that such terms will not appear in the model to be discussed below. 
Concerning the LEC's $C_{19}, C_{20}, C_{21}$, the following expressions are obtained,

$$
\begin{aligned}
& F_{0}^{-2} C_{19}=\frac{c_{m}^{2}}{M_{8}^{4}}\left(\stackrel{\circ}{g}_{m}+\frac{c c_{m}}{M_{8}^{2}}\right)+d^{\prime} \frac{c_{m}}{M_{8}^{2}} \\
& F_{0}^{-2} C_{20}=\frac{c_{m}^{2}}{M_{8}^{4}}\left(-\frac{2}{3} \stackrel{\circ}{g}_{m}+\stackrel{\circ}{m}_{m}-\frac{c c_{m}}{M_{8}^{2}}\right)+\frac{c_{m} \tilde{c}_{m}}{M_{0}^{2} M_{8}^{2}}\left(\stackrel{\circ}{f_{m}}+\frac{b c_{m}}{M_{8}^{2}}\right) \\
& \quad+\left(c^{\prime}-\frac{1}{3} d^{\prime}\right) \frac{c_{m}}{M_{8}^{2}}+b^{\prime} \frac{\tilde{c}_{m}}{M_{0}^{2}} \\
& F_{0}^{-2}\left(C_{20}+3 C_{21}\right)=-\frac{c_{m}^{2}}{3 M_{8}^{4}}\left(\stackrel{\circ}{m}_{m}+\frac{c c_{m}}{M_{8}^{2}}\right)+\frac{3 \tilde{c}_{m}^{2}}{M_{0}^{4}}\left(\stackrel{\circ}{h}_{m}+\frac{a \tilde{c}_{m}}{M_{0}^{2}}\right) \\
& \quad+\left(3 a^{\prime}+b^{\prime}\right) \frac{\tilde{c}_{m}}{M_{0}^{2}}-d^{\prime} \frac{c_{m}}{3 M_{8}^{2}} .
\end{aligned}
$$

\subsection{Parameters of the linear sigma-model}

In the $S U(3)$ linear sigma-model, one first encodes the scalar nonet and the pseudoscalar nonet into a complex $3 \times 3$ matrix $\Sigma$, which, under a chiral transformation transforms as,

$$
\Sigma \rightarrow g_{R} \Sigma g_{L}^{\dagger}
$$

and the lagrangian is assumed to be renormalizable. In the chiral limit, the most general renormalizable lagrangian, invariant under the chiral group (except for the $U_{A}(1)$ subgroup) contains four parameters,

$$
\begin{aligned}
& \mathcal{L}=\frac{1}{2}\left\langle\partial_{\mu} \Sigma \partial^{\mu} \Sigma^{\dagger}\right\rangle-\frac{1}{2} \mu^{2}\left\langle\Sigma \Sigma^{\dagger}\right\rangle-\lambda\left\langle\left(\Sigma \Sigma^{\dagger}\right)^{2}\right\rangle \\
& -\lambda^{\prime}\left\langle\Sigma \Sigma^{\dagger}\right\rangle^{2}+\beta\left(\operatorname{det}(\Sigma)+\operatorname{det}\left(\Sigma^{\dagger}\right)\right) .
\end{aligned}
$$

This lagrangian was reconsidered recently by several authors [15] 29] [30] 31] and we use the same notations as in ref. 31. Suitable choices of the parameters $\mu^{2}, \lambda, \lambda^{\prime}, \beta$ ensure that spontaneous chiral symmetry breaking occurs, i.e. that the potential is minimised for a non-zero value of the vacuum expectation value of the $\Sigma$ matrix,

$$
\bar{\Sigma}=v I_{d}
$$

(which we take to be diagonal, assuming that no spontaneous breaking of flavour symmetry occurs). Values of $v$ which correspond to extremums of the potential are solutions of the cubic equation,

$$
v\left(\left(4 \lambda+12 \lambda^{\prime}\right) v^{2}-2 \beta v+\mu^{2}\right)=0,
$$

which has real solutions $v \neq 0$ provided the parameters satisfy

$$
\beta^{2} \geq \mu^{2}\left(4 \lambda+12 \lambda^{\prime}\right)
$$


Next, expanding around the minimum we find that the pseudoscalars are massless, except for the singlet, $\eta_{0}$ which mass is proportional to $\beta$,

$$
M_{\eta_{0}}^{2}=6 \beta v
$$

We can also read off the expressions for the singlet and octet scalar meson masses in the chiral limit, $M_{0}$ and $M_{8}$,

$$
M_{8}^{2}=8 \lambda v^{2}+4 \beta v, \quad M_{0}^{2}=8\left(\lambda+3 \lambda^{\prime}\right) v^{2}-2 \beta v .
$$

Stability of the vacuum requires that these squared masses be positive. Using eqs. 3.18) (3.20) and (3.21) we can trade the original four parameters of the lagrangian (3.16) for the more physically relevant ones $v, M_{\eta_{0}}, M_{0}$ and $M_{8}$.

In QCD, chiral symmetry is broken by the light quark masses. We will make the simplifying assumption, at the level of the sigma-model, to consider symmetry breaking to linear order in the quark masses but we accept all the terms which are soft (i.e. of dimensionality strictly smaller than four). Under these assumptions the most general symmetry breaking sector has, again, four parameters,

$$
\begin{aligned}
& \mathcal{L}_{S B}=\gamma_{0}\left\langle\Sigma \chi^{\dagger}\right\rangle+\gamma_{1}\left\langle\Sigma^{-1} \chi\right\rangle \operatorname{det}(\Sigma)+\gamma_{2}\left\langle\Sigma \Sigma^{\dagger} \Sigma \chi^{\dagger}\right\rangle \\
& \quad+\gamma_{3}\left\langle\Sigma \Sigma^{\dagger}\right\rangle\left\langle\Sigma \chi^{\dagger}\right\rangle+\text { h.c. }
\end{aligned}
$$

Usually, only the first term is considered. This increased phenomenological flexibility will allow us to obtain better fits of the scalar nonet masses. While more general, this lagrangian will prove nevertheless to be reasonably constraining. We note that only the first two terms are renormalizable in the strict sense that the counterterms are exactly of the same form[17]. The last two terms generate counterterms which are of higher order in the scalar source $\chi$. Consistent with the assumption of not including further terms quadratic in the quark mass matrix at this level, we will compute the scalar meson masses and the vacuum expectation values at linear order as well. We have checked this approximation by comparing with exactly calculated masses.

The connection with the representation of the scalar fields used in sec.3.1 is performed by making a change of variables 32

$$
\Sigma=\exp \left(i \sqrt{\frac{2}{3}} \frac{\eta_{0}}{F_{0}}\right) u H u
$$

with

$$
u=\exp \left(i \sum_{1}^{8} \frac{\lambda_{a} \pi_{a}}{2 F_{0}}\right), \quad H=\left(v+\frac{S_{0}}{\sqrt{3}}\right) I_{d}+S .
$$

This change of variable makes sense only if $F_{0} \neq 0$, i.e. when chiral symmetry is spontaneously broken. The kinetic energy part of the linear sigma-model lagrangian 
gets transformed into

$$
\begin{aligned}
\mathcal{L}_{k i n}= & \frac{1}{2} \partial_{\mu} S_{0} \partial^{\mu} S_{0}+\frac{1}{2}\left\langle\nabla_{\mu} S \nabla^{\mu} S\right\rangle+\frac{1}{8}\left\langle\left\{u_{\mu}, H\right\}^{2}\right\rangle+\frac{1}{3 F_{0}^{2}} \partial_{\mu} \eta_{0} \partial^{\mu} \eta_{0}\left\langle H^{2}\right\rangle \\
& -\sqrt{\frac{2}{3}} \frac{\partial_{\mu} \eta_{0}}{F_{0}}\left\langle H^{2} u^{\mu}\right\rangle
\end{aligned}
$$

with

$$
\nabla_{\mu} S=\partial_{\mu} S+\left[\Gamma_{\mu}, S\right], \Gamma_{\mu}=\frac{1}{2}\left(u^{\dagger} \partial_{\mu} u+u \partial_{\mu} u^{\dagger}\right), u_{\mu}=i\left(u^{\dagger} \partial_{\mu} u-u \partial_{\mu} u^{\dagger}\right) .
$$

Replacing $H$ by its expression above (3.24) one identifies $v$ with the pion decay constant in the chiral limit $F_{0}$ and one obtains a prediction for the two couplings $c_{d}$ and $\tilde{c}_{d}$,

$$
v^{2}=\frac{F_{0}^{2}}{2}, \quad c_{d}=v, \quad \tilde{c}_{d}=\frac{v}{\sqrt{3}} .
$$

The symmetry breaking lagrangian, expressed in terms of the new fields reads

$$
\begin{aligned}
\mathcal{L}_{S B}= & \left\langle\left(\gamma_{0} H+\gamma_{2} H^{3}+\gamma_{3}\langle H\rangle^{2} H\right)\left(\chi_{+} \cos \sqrt{\frac{2}{3}} \frac{\eta_{0}}{F_{0}}-i \chi_{-} \sin \sqrt{\frac{2}{3}} \frac{\eta_{0}}{F_{0}}\right)\right\rangle \\
& +\gamma_{1} \operatorname{det} H\left\langle H^{-1}\left(\chi_{+} \cos \sqrt{\frac{8}{3}} \frac{\eta_{0}}{F_{0}}+i \chi_{-} \sin \sqrt{\frac{8}{3}} \frac{\eta_{0}}{F_{0}}\right)\right\rangle
\end{aligned}
$$

In this sector, we obtain one relation among the four parameters $\gamma_{i}$ from the requirement that the coefficient of the chiral lagrangian term $\left\langle\chi_{+}\right\rangle$be correctly normalized to $F_{0}^{2} / 4$ and we can express two other parameters in terms of the meson-source couplings $c_{m}$ and $\tilde{c}_{m}$. This gives the relations

$$
\begin{aligned}
& \gamma_{0}=\frac{3}{4} v+\frac{1}{6} c_{m}-\frac{2}{\sqrt{3}} \tilde{c}_{m}+\gamma_{3} v^{2} \\
& \gamma_{1} v=-\frac{1}{3}\left(c_{m}-\sqrt{3} \tilde{c}_{m}\right)-2 \gamma_{3} v^{2} \\
& \gamma_{2} v^{2}=-\frac{1}{4} v+\frac{1}{6} c_{m}+\frac{1}{\sqrt{3}} \tilde{c}_{m}-2 \gamma_{3} v^{2} .
\end{aligned}
$$

In this sector, we can take $c_{m}, \tilde{c}_{m}$ and $\gamma_{3}$ as arbitrary parameters.

In this model, we can now express the many parameters that appeared in the general scalar lagrangian discussed above. Firstly, for the terms coupling one scalar field to two scalar sources we have simply $a^{\prime}=b^{\prime}=c^{\prime}=d^{\prime}=0$. For the trilinear couplings of resonances, one obtains the relations

$$
\begin{aligned}
a & =-\frac{1}{2 \sqrt{3} v}\left(M_{0}^{2}+\frac{1}{9} M_{\eta_{0}}^{2}\right) \\
b & =-\frac{1}{2 \sqrt{3} v}\left(M_{0}^{2}+2 M_{8}^{2}-\frac{2}{3} M_{\eta_{0}}^{2}\right) \\
c & =-\frac{1}{2 v}\left(M_{8}^{2}-\frac{8}{9} M_{\eta_{0}}^{2}\right) .
\end{aligned}
$$


Finally, the parameters $g_{m}, h_{m}, e_{m}, f_{m}$ which control linear symmetry breaking of the scalar meson masses (see eqs.(3.12), (3.13)) obey the following relations,

$$
\begin{aligned}
& g_{m}=\frac{4}{3 v}\left(\sqrt{3} \tilde{c}_{m}-c_{m}+c_{m} \frac{M_{\eta_{0}}^{2}}{M_{8}^{2}}\right)-\frac{3}{4}-8 \gamma_{3} v \\
& \sqrt{3} f_{m}=\frac{1}{v}\left(\sqrt{3} \tilde{c}_{m}-c_{m} \frac{M_{0}^{2}}{M_{8}^{2}}\right)-\frac{9}{8}+\frac{1}{2} g_{m} \\
& e_{m}=\frac{M_{\eta_{0}}^{2}-3 M_{8}^{2}}{9 M_{0}^{2} v}\left(\sqrt{3} \tilde{c}_{m}-c_{m} \frac{M_{0}^{2}}{M_{8}^{2}}\right)-\frac{3}{16}-\frac{1}{4} g_{m} \\
& h_{m}=-\frac{M_{\eta_{0}}^{2}}{18 M_{0}^{2} v}\left(\sqrt{3} \tilde{c}_{m}-c_{m} \frac{M_{0}^{2}}{M_{8}^{2}}\right)-\frac{9}{32}-\frac{1}{24} g_{m} .
\end{aligned}
$$

Making use of the relations (3.11) we can express the three $O\left(p^{6}\right)$ LEC's $C_{19}, C_{20}, C_{21}$ discussed above in terms of the parameters of the linear sigma-model. We are also interested in the prediction for the OZI suppressed $O\left(p^{6}\right)$ LEC $C_{16}$ which participates in the flavour variation of the order parameter $F_{\pi}$ as discussed above. After a small calculation, the following expression is obtained,

$$
\begin{aligned}
F_{0}^{-2} C_{16}= & \frac{1}{54}\left(2 c_{m}+10 \sqrt{3} \tilde{c}_{m}-9 v\right)\left(\frac{1}{M_{0}^{2}}-\frac{1}{M_{8}^{2}}\right)\left(\frac{2 c_{m}}{M_{8}^{2}}+\frac{\sqrt{3} \tilde{c}_{m}}{M_{0}^{2}}\right) \\
& +\frac{\gamma_{3} v^{2}}{9}\left(\frac{2 \sqrt{3} \tilde{c}_{m}}{M_{0}^{2}}\left(\frac{1}{M_{0}^{2}}+\frac{2}{M_{8}^{2}}\right)-\frac{4 c_{m}}{M_{8}^{2}}\left(\frac{2}{M_{0}^{2}}-\frac{5}{M_{8}^{2}}\right)\right) \\
& +\frac{M_{\eta_{0}}^{2}}{54 M_{0}^{2} M_{8}^{2}}\left(\frac{2 c_{m}}{M_{8}^{2}}+\frac{\sqrt{3} \tilde{c}_{m}}{M_{0}^{2}}\right)\left(2 c_{m}\left(1-2 \frac{M_{0}^{2}}{M_{8}^{2}}\right)-\sqrt{3} c_{m} \frac{M_{8}^{2}}{M_{0}^{2}}\right) .
\end{aligned}
$$

\subsection{Phenomenological applications}

Altogether, the sigma-model as considered here has six independent parameters (apart from $F_{0}$ ): three chiral limit masses $M_{0}, M_{8}$ and $M_{\eta_{0}}$ and three couplings to the scalar source $c_{m}, \tilde{c}_{m}$ and $\gamma_{3}$. The parameter $M_{\eta_{0}}$, i.e. the mass of the $\eta^{\prime}$ in the chiral limit can be estimated to be [4,

$$
M_{\eta_{0}} \simeq 0.8 \mathrm{GeV}
$$

Let us now discuss the determination of the remaining five parameters. A priori, one expects to be able to reproduce exactly the four independent masses $\left(M_{a_{0}}, M_{\kappa_{0}}\right.$, $\left.M_{\sigma_{0}}, M_{f_{0}}\right)$ in the scalar nonet and, as an additional constraint a natural choice would be to enforce the super-convergence relation (2.15) which implies here $c_{m}=\sqrt{3} \tilde{c}_{m}$. In practice, however, because of non-linearities, this choice strongly restricts the possible range of masses for the $\kappa_{0}$. This is an indication that this model should be considered as a toy model rather than a really physically meaningful one. Instead, we did not try to impose the super-convergence relation and use a constraint from the pseudo-scalar sector, the ratio $F_{K} / F_{\pi}$ which allows for a fairly broad range of 
values for $M_{\kappa_{0}}$. Because of the specific parametrisation of the mass matrix, eq.(3.13) its entries get evaluated as follows in terms of physical masses,

$$
\mathcal{M}_{22}=\frac{4 M_{\kappa_{0}}^{2}-M_{a_{0}}^{2}}{3}, \quad \mathcal{M}_{11}=M_{\sigma_{0}}^{2}+M_{f_{0}}^{2}-\mathcal{M}_{22}
$$

and, using the determinant,

$$
\mathcal{M}_{12}= \pm \sqrt{-\left(\mathcal{M}_{22}-M_{\sigma_{0}}^{2}\right)\left(\mathcal{M}_{22}-M_{f_{0}}^{2}\right)}
$$

We observe that there are two possible sign assignments. Reality of $\mathcal{M}_{12}$ requires that the following inequality be satisfied

$$
\frac{M_{a_{0}}^{2}+3 M_{\sigma_{0}}^{2}}{4} \leq M_{\kappa_{0}}^{2} \leq \frac{M_{a_{0}}^{2}+3 M_{f_{0}}^{2}}{4}
$$

As shown in [33, this inequality holds in a model independent way if symmetry breaking is assumed to be linear. From the expression of the mass matrix (3.13) it is then easy to see that the scalar singlet chiral mass $M_{0}^{2}$ satisfies a quadratic equation

$$
M_{0}^{4}-B M_{0}^{2}+C M_{\eta_{0}}^{2}=0
$$

with

$$
\begin{aligned}
& B=\mathcal{M}_{11}-\frac{9}{8}\left(2 m_{K}^{2}+m_{\pi}^{2}\right)\left(1-\frac{1}{27} \frac{M_{\kappa_{0}}^{2}-M_{a_{0}}^{2}}{m_{K}^{2}-m_{\pi}^{2}}\right) \\
& C=\frac{1}{4}\left(2 m_{K}^{2}+m_{\pi}^{2}\right)\left(1+\frac{1}{9} \frac{M_{\kappa_{0}}^{2}-M_{a_{0}}^{2}}{m_{K}^{2}-m_{\pi}^{2}}+\frac{\sqrt{2}}{3} \frac{\mathcal{M}_{12}}{m_{K}^{2}-m_{\pi}^{2}}\right) .
\end{aligned}
$$

Real solutions exist provided

$$
B^{2}-4 C M_{\eta_{0}}^{2} \geq 0
$$

and it must further be checked that at least one solution is positive. This puts further constraints on the allowed values of the scalar meson masses in this model. Once $M_{0}^{2}$ is determined, the other parameters are easily evaluated. For instance $M_{8}^{2}$ is given by,

$$
\begin{aligned}
M_{8}^{2} & =\frac{1}{1+\frac{6 C}{M_{0}^{2}}}\left[M_{a_{0}}^{2}+\frac{2 C M_{\eta_{0}}^{2}}{M_{0}^{2}}-\frac{3}{4}\left(2 m_{K}^{2}+m_{\pi}^{2}\right)\right. \\
& \left.+\frac{1}{4}\left(2 m_{K}^{2}-3 m_{\pi}^{2}\right) \frac{M_{\kappa_{0}}^{2}-M_{a_{0}}^{2}}{m_{K}^{2}-m_{\pi}^{2}}\right]
\end{aligned}
$$

Next, using as experimental input $M_{\kappa_{0}}^{2}-M_{a_{0}}^{2}$ and $\mathcal{M}_{12}$ together with eqs.(3.12), (3.13) and (3.31) ) gives two linear equations for the three quantities $c_{m} / v, \tilde{c}_{m} / v$ and 
$\gamma_{3} v$. We will use as an additional constraint, the ratio $F_{K} / F_{\pi}$, which determines $c_{m} / v$ from the relation,

$$
\frac{c_{m}}{v}=\frac{M_{8}^{2}}{2 F_{\pi}} \frac{F_{K}-F_{\pi}}{m_{K}^{2}-m_{\pi}^{2}}
$$

Finally, there remains to determine $v=F_{0} / \sqrt{2}$. To linear order in the quark masses, $v$ is given by the following expression

$$
v=\frac{F_{\pi}}{\sqrt{2}}\left[1-\frac{2}{3} m_{\pi}^{2}\left(\frac{2 c_{m} / v}{M_{8}^{2}}+\frac{\sqrt{3} \tilde{c}_{m} / v}{M_{0}^{2}}\right)-\frac{4}{3} m_{K}^{2}\left(\frac{-c_{m} / v}{M_{8}^{2}}+\frac{\sqrt{3} \tilde{c}_{m} / v}{M_{0}^{2}}\right)\right] .
$$

This expression can be recovered in two different ways: one can either use CHPT together with the expressions (3.3) for $L_{4}$ and $L_{5}$ or write down the equations for the vacuum expectation values in the presence of quark masses and solve these equations to linear order in the quark masses.

\subsubsection{Light $\sigma$ meson}

The existence of a very broad scalar resonance in $\pi \pi$ scattering with $M \simeq \Gamma \simeq$ $0.5-0.6 \mathrm{GeV}$ is by now well established since the work of Basdevant Froggatt and Petersen[18], in which the whole set of constraints from unitarity, analyticity, and crossing symmetry has been implemented (see ref.35 for a recent survey and the particle data book[5] for a complete list of references). What is unclear is whether this state should be interpreted as a physical light scalar resonance. Recently, Black et al. 20] have proposed arguments based on perturbative unitarity favouring the existence of a light $\sigma$ and also, of a light $\kappa$ meson. According to the inequality (3.36), if the sigma meson is light then one must have $M_{\kappa_{0}} \leq M_{a_{0}}$ unless our assumption of linearity in the quark masses fails. Recently, Törnqvist [31] (similar fits were also discussed in ref. 15]) has attempted to accommodate in the linear sigma-model a scalar meson multiplet with a light sigma and a heavy $\kappa$. Such fits fail to obtain the $f_{0}(980)$ at the correct mass and therefore would not correctly reproduce the $\pi \pi$ phase-shifts around $1 \mathrm{GeV}$, even if one-loop corrections are included 34].

Let us now assume that a light sigma meson exists, e.g. $M_{\sigma_{0}} \simeq 0.6 \mathrm{GeV}$, and discuss the consequences. We take otherwise $M_{a_{0}}=0.983 \mathrm{GeV}$, and $M_{f_{0}}=0.980$ $\mathrm{GeV}$ from experiment and $M_{\kappa_{0}}=0.9 \mathrm{GeV}$ as proposed in ref. [20]. From this input one easily calculates the parameters $A$ and $B$ in the quadratic equation (3.37),

$$
A=0.024 \mathrm{GeV}^{2} \quad B=0.042 \mathrm{GeV}^{2} .
$$

There results that no real solution to the equation for $M_{0}^{2}$ exists unless $M_{\eta_{0}} \leq 0.06$ $\mathrm{GeV}$, which appears as an absurdly small value. Reality and positivity of $M_{0}^{2}$ (and $M_{8}^{2}$ ) are necessary conditions for the existence of a stable minimum of the potential, with spontaneous chiral symmetry breaking. One must thus conclude that with such input scalar meson masses $N_{F}=3$ chiral symmetry is not spontaneously broken in 


\begin{tabular}{|c||c|c|c|c|c|c|}
\hline$M_{\kappa_{0}}$ & $M_{0}$ & $M_{8}$ & $c_{m}$ & $\sqrt{3} \tilde{c}_{m}$ & $\gamma_{3}$ & $\sqrt{2} v$ \\
\hline 1.10 & 1.17 & 0.85 & 0.023 & 0.040 & 0.54 & 0.092 \\
1.20 & 1.05 & 0.93 & 0.028 & 0.027 & 0.33 & 0.094 \\
1.30 & 0.87 & 0.90 & 0.023 & 0.034 & 1.56 & 0.081 \\
1.33 & 0.79 & 0.85 & 0.017 & 0.037 & 2.79 & 0.068 \\
1.35 & 0.72 & 0.80 & 0.011 & 0.033 & 4.82 & 0.051 \\
\hline
\end{tabular}

Table 3: Determination of parameters in the case of a heavy scalar nonet $M_{\sigma_{0}}=0.980$, $M_{f_{0}}=1.5$ for several values of $M_{\kappa_{0}}$. All entries are in $\mathrm{GeV}$ except $\gamma_{3}$ which is in $\mathrm{GeV}^{-1}$.

the linear sigma-model. The same conclusion holds if one picks up larger values for $M_{\kappa_{0}}$ : using the set of parameters determined in ref. [15] for $M_{\sigma}=0.4$ and $M_{\sigma}=0.6$ $\mathrm{GeV}$ one verifies that the equation for the vacuum expectation value $v$ eq.(3.18) has no real solution $v \neq 0$. A better situation prevails if we accept smaller values for $M_{\kappa_{0}}$. If we take, for instance, $M_{\kappa_{0}}=0.750 \mathrm{GeV}$, which is the smallest value allowed by the inequality(3.36), then, eq.(3.37) has real solutions provided $M_{\eta_{0}} \leq 0.62 \mathrm{GeV}$, which is still reasonable. A problem remains, however, that being close to a situation where chiral symmetry is unbroken for $N_{F}=3$, while it is in general broken for $N_{F}=2$, the expansion in the strange quark mass is likely to be non-converging. Indeed, if we try to compute $F_{0}$ with, say, $M_{\eta_{0}}=0.50 \mathrm{GeV}$, using our linear expansion formula (3.42), we find $F_{0} \simeq 28 \mathrm{MeV}$, which is very small compared to $F_{\pi}$, suggesting that the expansion in $m_{s}$ is not perturbative in this case.

\subsubsection{Heavy $\sigma$ meson}

Let us now consider the scenario of a heavy sigma meson, i.e. we identify the sigma meson with the well established resonance $f_{0}(980)$ and we identify the heavier $I=0$ member of the nonet with the $f_{0}(1500)$ (see [24). With these assignments, we find that a solution of eq.(3.37) with real $M_{0}^{2}$ exists in the range

$$
M_{\kappa_{0}} \leq 1.37 \mathrm{GeV},
$$

taking $M_{\eta_{0}}=0.8 \mathrm{GeV}$ and the negative sign for $\mathcal{M}_{12}$ (see (3.35)). This means that we cannot exactly reproduce the experimental mass of the $K_{0}^{*}(1430)$ but we can get reasonably close. We will eventually allow $M_{\kappa_{0}}$ to vary somewhat away from the experimental result. Numerical values of the parameters $M_{0}, M_{8}, c_{m}, \tilde{c}_{m}, \gamma_{3}$ as well as $v$ determined for several values of $M_{\kappa_{0}}$ are collected in table (3). We note that the situation with a minimal symmetry breaking lagrangian (i.e. $\gamma_{1}=\gamma_{2}=\gamma_{3}=0$ ) is close to the case when $M_{\kappa_{0}}=1.20$ in the table. In this case, one has $c_{m}=$ $\sqrt{3} \tilde{c}_{m}=v / 2$. The value of $F_{0}=\sqrt{2} v$ remains reasonable close to $F_{\pi}$ except when $M_{\kappa_{0}}$ gets very near to its upper bound. The coupling $c_{m}$ comes out smaller than

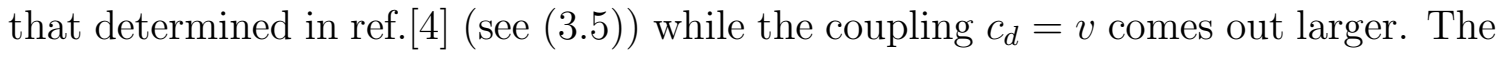
coupling $c_{d}$ controls the decay width $a_{0} \rightarrow \pi \eta$, which tends to be too large in this 


\begin{tabular}{|c||c|c|c||c|c|}
\hline$M_{\kappa_{0}}$ & $10^{3} L_{5}$ & $10^{3} L_{7}$ & $10^{3} L_{8}$ & $10^{5} C_{19}$ & $10^{5} C_{20}$ \\
\hline 1.10 & 2.06 & -0.22 & 0.36 & -0.17 & -0.05 \\
1.20 & 2.15 & -0.47 & 0.45 & -0.34 & 0.07 \\
1.30 & 1.61 & -0.62 & 0.32 & -0.45 & 0.12 \\
1.33 & 1.13 & -0.51 & 0.20 & -0.38 & 0.08 \\
1.35 & 0.64 & -0.31 & 0.10 & -0.24 & 0.04 \\
\hline
\end{tabular}

Table 4: Numerical values of some $O\left(p^{4}\right)$ and $O\left(p^{6}\right)$ LEC's from the sigma-model with a heavy sigma.

model (accepting that the experimental value is $\Gamma \simeq 60 \mathrm{MeV}$, which is subject to some debate[5]), the result in this respect improves as $M_{\kappa_{0}}$ gets larger.

Once these couplings are known it is easy to determine the LEC's with the formulas given above. Numerical values for the LEC's dominated by the scalar mesons and not suppressed in the large $N_{c}$ limit are shown in table (4). We have also included $L_{7}$, which is assumed to be saturated by the $\eta_{0}$, computed from the expression [4,

$$
L_{7}=-\frac{\tilde{d}_{m}^{2}}{2 M_{\eta_{0}}^{2}},
$$

where the coupling $\tilde{d}_{m}$ of the $\eta_{0}$ field to the pseudo-scalar current has the following expression in the present version of the linear sigma-model,

$$
\tilde{d}_{m}=-\frac{F_{0}}{2 \sqrt{6}}\left(1+\frac{2}{v}\left(c_{m}-\sqrt{3} \tilde{c}_{m}\right)+12 \gamma_{3} v\right) .
$$

The fact that $L_{5}$ does not remain constant when $M_{\kappa_{0}}$ increases is a reflection of the effect of non-linearities in $m_{s}$. The values of the LEC's may be compared with the ones determined at $O\left(p^{4}\right)$ from experimental data [1]

$$
10^{3} L_{5}^{r}\left(m_{\eta}\right)=2.2 \pm 0.5, \quad 10^{3} L_{7}=-0.4 \pm 0.15, \quad 10^{3} L_{8}^{r}\left(m_{\eta}\right)=1.1 \pm 0.3 .
$$

While the prediction for $L_{7}$ is acceptable, that for $L_{8}$ is too small. This is related to the small size of the meson to source coupling $c_{m}$ predicted by the model. Still, the order of magnitude is correct except, perhaps, for the very last line in the table. The LEC's were recently re-determined in an $O\left(p^{6}\right)$ analysis of the experimental data [36] and this brings the values somewhat down in magnitude: $10^{3} L_{5}^{r}\left(m_{\eta}\right)=$ $1.45 \pm 0.12,10^{3} L_{7}=-0.31 \pm 0.15,10^{3} L_{8}^{r}\left(m_{\eta}\right)=0.68 \pm 0.18$. A resonance-saturated estimate for the $O\left(p^{6}\right)$ coupling $C_{19}$ was provided in ref.[12]. The value that we obtain is significantly smaller, by a factor $3-4$. One difference in the estimates is that we include the effect of cubic interaction terms, but the numerical influence of these terms turns out to be unimportant. The main reason for our smaller result is, again, the fact that the coupling $c_{m}$ is smaller here. 


\begin{tabular}{|c||c|c||c|c|}
\hline$M_{\kappa_{0}}$ & $10^{3} L_{4}$ & $10^{3} L_{6}$ & $10^{5} C_{16}$ & $10^{5}\left(C_{20}+3 C_{21}\right)$ \\
\hline 1.10 & -0.06 & 0.07 & 0.19 & 0.02 \\
1.20 & -0.16 & -0.04 & 0.08 & 0.05 \\
1.30 & 0.33 & 0.15 & 0.21 & 0.08 \\
1.33 & 0.56 & 0.28 & 0.36 & 0.15 \\
1.35 & 0.55 & 0.31 & 0.41 & 0.27 \\
\hline
\end{tabular}

Table 5: Numerical values of OZI suppressed $O\left(p^{4}\right)$ and $O\left(p^{6}\right)$ LEC's from the sigmamodel with a heavy sigma.

Let us now return to the OZI suppressed coupling constants $L_{4}$ and $L_{6}$ and their $O\left(p^{6}\right)$ counterparts $C_{16}$ and $C_{20}+3 C_{21}$. The predictions from the linear sigma-model are collected in table (5). The values and signs of $L_{4}, L_{6}$ are seen to depend very much on the mass of $\kappa_{0}$. They both approximately vanish when $M_{\kappa_{0}} \simeq 1.24 \mathrm{GeV}$. This is the point of minimal OZI violation. As one pushes the $\kappa_{0}$ to higher masses, as required by experiment, then the pattern of OZI violation is not unlike the one found on the basis of sum rules. Both $L_{4}$ and $L_{6}$ become positive, and the orders of magnitudes seem to be in agreement with the discussion based on sum rules. There is even a relatively good agreement as far as $L_{4}$ and $C_{16}$ are concerned.

\section{Conclusions}

In this paper, we have pursued an investigation on the evolution of order parameters of the QCD chiral vacuum as one increases the number of massless flavours, as can be inferred from experimental data. An information on this evolution is contained in the $O\left(p^{4}\right)$ coupling constants $L_{4}$ and $L_{6}$ which control how $F_{\pi}$ and $\langle\bar{u} u\rangle$ respectively vary from the $S U(2)$ chiral limit with $m_{s} \simeq 200 \mathrm{MeV}$ to the $S U(3)$ chiral limit. Estimates for the couplings $L_{4}$ and $L_{6}$ are based on the scalar form factors of the pion and the Kaon which are reconstructed from experimental S-wave scattering data modulo some assumptions. We have investigated the $O\left(m_{s}\right)$ corrections to our previous results. These have two origins: a) the normalization of the form factors at the origin must include the $O\left(p^{4}\right)$ contributions and b) the chiral expansions (for $F_{\pi}$ and $\left.\Pi_{6}(0)\right)$ must be pursued up to order $p^{6}$.

These corrections seem not to affect in a significant way the results obtained previously. In particular there is no sign that these corrections go in the sense of decreasing the values of $L_{4}$ and $L_{6}$ to make them compatible with the naive large $N_{c}$ expectation. This conclusion, however, only holds provided one makes the assumption that the chiral expansion is reasonable, in other terms, that the $O\left(p^{6}\right)$ contribution (say in the expansion of $\left.\Pi_{6}(0)\right)$ is not larger than the $O\left(p^{4}\right)$ contribution. Modulo this assumption, one also obtains from this analysis an estimate of the $O\left(p^{6}\right)$ coupling constant $C_{16}$ and the combination $C_{20}+3 C_{21}$. Influence of the values of $L_{4}$, 
$L_{6}$ on the convergence rate of the chiral expansion for various observables was studied very recently in [37]. Because of such assumptions which enter into the calculation, the error bars on our coupling constant estimates must be considered as educated guesses. Our results are compatible with a tendency towards chiral restoration upon increasing $N_{F}$ from $N_{F}=2$ to $N_{F}=3$ which is significant, particularly so for the quark condensate (in agreement with the arguments of [6]), for which the decrease by a factor of approximately two found in ref.[7] is confirmed. This behaviour of the quark condensate is in qualitative agreement with the one obtained from the instanton liquid model 38]. It would be also interesting to compare this result with unquenched lattice simulations with $N_{F}=3$. Unfortunately, such simulations are not available yet, but will exist in the near future.

We also discussed some predictions from the linear sigma-model, considered as a simple toy model. One application is to gauge the possible importance of selfcouplings of resonances (which are present in the model) in resonance saturation estimates of the $O\left(p^{6}\right)$ LEC's. Furthermore, the model provides a connection between simply the spectrum of the scalars and the evolution of chiral order parameters with $N_{F}$. In order to improve the ability of the model to reproduce experimental masses, the symmetry breaking sector was made more general than usually done, but still linear in the quark mass matrix. We have considered two possibilities for the light scalar nonet:

a)The nonet contains a light $\sigma$ and a light $\kappa$. In this case, we find that chiral symmetry tends to be restored already at $N_{F}=3$ or, at least, for a value of $N_{F}$ somewhat too small, making the chiral expansion in $m_{s}$ unreliable.

b) The nonet is composed of the resonances $a_{0}(980), f_{0}(980), K_{0}^{*}(1430)$ and $f_{0}(1500)$ (which are well established). In this case, we find that we need only a small amount of nonlinear effects to exactly reproduce the $K_{0}^{*}(1430)$ mass, and $S U(3)$ chiral symmetry is realised in the Goldstone mode. Concerning $L_{4}$ and $L_{6}$, we find that they become different from zero as one increases the mass of the $K_{0}^{*}(1430)$ towards its experimental value. The signs and orders of magnitude, then, are compatible with the preceding analysis. The same is true of the related $O\left(p^{6}\right)$ constants.

\section{Acknowledgments}

The author thanks B. Ananthanarayan for drawing his attention to ref. 24 and Jan Stern for useful remarks. This work is supported in part by funds provided by the U.S. Department of Energy (D.O.E.) under cooperative research agreement \#DF-FC02-94ER40818 and by the EURODAPHNE network EEC contract \#TMRERBFMRX-CT980169. 


\section{A. The correlation function $\Pi_{6}(s)$ to $O\left(p^{6}\right)$}

At chiral order $p^{4}$, a simple calculation gives the correlation function $\Pi_{6}(s)$,

$$
\Pi_{6}(s)=2 \bar{J}_{K}(s)+\frac{4}{9} \bar{J}_{\eta}(s)+64 L_{6}^{r}-\frac{1}{16 \pi^{2}}\left[2 L_{K}+\frac{4}{9} L_{\eta}+\frac{22}{9}\right],
$$

where $\bar{J}_{P}(s)$ is the one-loop function defined as to vanish for $s=0$,

$$
\bar{J}_{P}(s)=\frac{1}{16 \pi^{2}}\left(\sigma_{P} \log \frac{\sigma_{P}-1}{\sigma_{P}+1}+2\right), \sigma_{P}=\sqrt{1-\frac{4 M_{P}^{2}}{s}} .
$$

In all the formulas $M_{P}^{2}$ stands not for the physical pseudo-scalar meson masses but for their lowest order chiral expansion,

$$
M_{\pi}^{2} \equiv 2 m B_{0}, \quad M_{K}^{2} \equiv\left(m+m_{s}\right) B_{0}, \quad M_{\eta}^{2} \equiv \frac{\left(2 m+4 m_{s}\right) B_{0}}{3} .
$$

The following simplified notation for logarithms was introduced

$$
L_{P}=\log \frac{M_{P}^{2}}{\mu^{2}} .
$$

At order $p^{6}$, one must compute the one-loop and two-loops diagrams shown in fig.1 and add the tree-level contributions from the $O\left(p^{6}\right)$ chiral lagrangian. The calculation is lengthy but not excessively difficult because of the absence of sunset-type diagrams in the present case. The result may be written as a sum of six terms:

$$
\Delta \Pi_{6}(s)=\Delta \Pi_{6}^{a}(s)+\Delta \Pi_{6}^{b}(s)+\Delta \Pi_{6}^{r e s c}(s)+A \frac{s}{F_{0}^{2}}+B+C .
$$

The first term encodes the $O\left(p^{4}\right)$ corrections to the pseudo-scalar meson masses in the one-loop functions,

$$
\Delta \Pi_{6}^{a}(s)=-s\left(2 \frac{\Delta M_{K}^{2}}{M_{K}^{2}} \frac{d \bar{J}_{K}(s)}{d s}+\frac{4}{9} \frac{\Delta M_{\eta}^{2}}{M_{\eta}^{2}} \frac{d \bar{J}_{\eta}(s)}{d s}\right) .
$$

These mass corrections are given by the following expressions $\mathbb{1}$,

$$
\begin{aligned}
& \Delta M_{K}^{2}=M_{K}^{2}\left[\frac{m B_{0}}{F_{0}^{2}}\left(-32 S_{6}-8 S_{8}+\frac{1}{72 \pi^{2}} L_{\eta}\right)\right. \\
& \left.+\frac{m_{s} B_{0}}{F_{0}^{2}}\left(-16 S_{6}-8 S_{8}+\frac{1}{36 \pi^{2}} L_{\eta}\right)\right]
\end{aligned}
$$

and

$$
\begin{aligned}
& \Delta M_{\eta}^{2}=M_{\eta}^{2}\left[\frac{m B_{0}}{F_{0}^{2}}\left(-32 S_{6}-\frac{16}{3} S_{8}-\frac{5}{48 \pi^{2}} L_{\pi}+\frac{1}{9 \pi^{2}} L_{K}-\frac{1}{144 \pi^{2}} L_{\eta}\right)\right. \\
& \left.+\frac{m_{s} B_{0}}{F_{0}^{2}}\left(-16 S_{6}-\frac{32}{3} S_{8}+\frac{1}{24 \pi^{2}} L_{\pi}+\frac{1}{18 \pi^{2}} L_{K}-\frac{1}{18 \pi^{2}} L_{\eta}\right)\right] \\
& +\frac{\left(m_{s}-m\right)^{2} B_{0}^{2}}{F_{0}^{2}}\left[\frac{128}{9} S_{7}+\frac{1}{108 \pi^{2}} L_{K}-\frac{1}{18 \pi^{2}} L_{\pi}\right]
\end{aligned}
$$


In these expressions, $S_{6}, S_{7}$ and $S_{8}$ denote the following combinations of $O\left(p^{4}\right)$ coupling constants,

$$
S_{6}=-2 L_{6}^{r}+L_{4}^{r}, S_{7}=3 L_{7}^{r}+L_{8}^{r}, S_{8}=-2 L_{8}^{r}+L_{5}^{r} .
$$

The second corrective term $\Delta \Pi_{6}^{b}(s)$ is generated by $O\left(p^{4}\right)$ corrections to the scalar form factors and has the following form,

$$
\begin{aligned}
& \Delta \Pi_{6}^{b}(s)=3 \bar{J}_{\pi}(s)\left[\frac{d \Delta M_{\pi}^{2}}{d m_{s} B_{0}}+\frac{s}{F_{0}^{2}}\left(8 L_{4}^{r}-\frac{1}{32 \pi^{2}}\left(L_{K}+1\right)\right)\right] \\
& +\bar{J}_{K}(s)\left[2 \frac{d \Delta M_{K}^{2}}{d m B_{0}}+2 \frac{d \Delta M_{K}^{2}}{d m_{s} B_{0}}+\frac{s}{F_{0}^{2}}\left(16 L_{5}^{r}+48 L_{4}^{r}\right.\right. \\
& \left.\left.-\frac{1}{32 \pi^{2}}\left(3 L_{\pi}+6 L_{K}+3 L_{\eta}+12\right)\right)\right]+\frac{1}{3} \bar{J}_{\eta}(s)\left[2 \frac{d \Delta M_{\eta}^{2}}{d m B_{0}}+\frac{d \Delta M_{\eta}^{2}}{d m_{s} B_{0}}\right. \\
& \left.+\frac{s}{F_{0}^{2}}\left(\frac{32}{3} L_{5}^{r}+40 L_{4}^{r}-\frac{9}{32 \pi^{2}}\left(L_{K}+1\right)\right)\right] .
\end{aligned}
$$

The derivatives of $\Delta M_{K}^{2}$ and $\Delta M_{\eta}^{2}$ are easily computed from the formulas above and for $\Delta M_{\pi}^{2}$ one has,

$$
\frac{d \Delta M_{\pi}^{2}}{m_{s} B_{0}}=m B_{0}\left(-32 S_{6}-\frac{1}{36 \pi^{2}}\left(L_{\eta}+1\right)\right) .
$$

The third corrective term $\Delta \prod_{6}^{r e s c}(s)$ is the rescattering contribution,

$$
\begin{aligned}
& \Delta \Pi_{6}^{r e s c}(s)=\frac{3 s}{2 F_{0}^{2}} \bar{J}_{K}(s)\left(\bar{J}_{\pi}(s)+\bar{J}_{K}(s)+\bar{J}_{\eta}(s)\right) \\
& +\frac{4}{3} \bar{J}_{\eta}(s)\left(\frac{\left(m+8 m_{s}\right) B_{0}}{27 F_{0}^{2}} \bar{J}_{\eta}(s)-\frac{\left(m+m_{s}\right) B_{0}}{F_{0}^{2}} \bar{J}_{K}(s)+\frac{m B_{0}}{F_{0}^{2}} \bar{J}_{\pi}(s)\right) .
\end{aligned}
$$

The last corrective term is a polynomial, linear in $s$. This term picks up contributions from four coupling constants of the $O\left(p^{6}\right)$ chiral lagrangian, $C_{39}^{r}, C_{20}^{r}, C_{21}^{r}$ and $C_{94}^{r}$ in the notation of [10]. The part proportional to $s$ is as follows

$$
\begin{aligned}
& A=64 C_{39}^{r}+\frac{1}{\pi^{2}}\left[\frac{3}{512 \pi^{2}} L_{K}\left(L_{\pi}+L_{K}+L_{\eta}\right)+L_{\pi}\left(\frac{3}{512 \pi^{2}}-\frac{3}{2} L_{4}^{r}\right)\right. \\
& +L_{K}\left(\frac{3}{128 \pi^{2}}-3 L_{4}^{r}-L_{5}^{r}\right)+L_{\eta}\left(\frac{3}{512 \pi^{2}}-\frac{5}{6} L_{4}^{r}-\frac{2}{9} L_{5}^{r}\right) \\
& \left.+\frac{9}{512 \pi^{2}}-\frac{16}{3} L_{4}^{r}-\frac{11}{9} L_{5}^{r}\right]
\end{aligned}
$$

The constant terms, finally are

$$
B=\frac{m B_{0}}{F_{0}^{2}}\left[32\left(8 C_{20}^{r}+48 C_{21}^{r}+C_{94}\right)-\frac{1}{864 \pi^{4}} L_{\eta}\left(-9 L_{\pi}+12 L_{K}-L_{\eta}\right)\right.
$$




$$
\begin{aligned}
& +\frac{L_{\pi}}{\pi^{2}}\left(12 S_{6}+\frac{1}{96 \pi^{2}}\right)+\frac{L_{K}}{\pi^{2}}\left(20 S_{6}+6 S_{8}-\frac{1}{96 \pi^{2}}\right) \\
& +\frac{L_{\eta}}{\pi^{2}}\left(4 S_{6}+\frac{8}{9} S_{8}-\frac{11}{5184 \pi^{2}}\right) \\
& \left.+\frac{1}{\pi^{2}}\left(\frac{206}{9} S_{6}-\frac{16}{27} S_{7}+\frac{124}{27} S_{8}+\frac{1}{5184 \pi^{2}}\right)\right] \\
& +\frac{m_{s} B_{0}}{F_{0}^{2}}\left[256\left(C_{20}^{r}+3 C_{21}^{r}\right)-\frac{1}{288 \pi^{4}} L_{\eta}\left(-L_{\eta}+5 L_{K}\right)\right. \\
& +\frac{L_{K}}{\pi^{2}}\left(16 S_{6}+6 S_{8}-\frac{1}{96 \pi^{2}}\right)+\frac{L_{\eta}}{9 \pi^{2}}\left(48 S_{6}+16 S_{7}+16 S_{8}-\frac{37}{576 \pi^{2}}\right) \\
& \left.+\frac{1}{\pi^{2}}\left(\frac{118}{9} S_{6}+\frac{16}{27} S_{7}+\frac{140}{27} S_{8}-\frac{19}{5184 \pi^{2}}\right)\right]
\end{aligned}
$$

and

$$
C=-\frac{1}{16 \pi^{2}}\left(2 \frac{\Delta M_{K}^{2}}{M_{K}^{2}}+\frac{4}{9} \frac{\Delta M_{\eta}^{2}}{M_{\eta}^{2}}\right)
$$

The terms proportional to the mass corrections $\Delta M_{K}^{2}$ and $\Delta M_{\eta}^{2}$ (see eq.(A.6) and (A.15) may eventually be absorbed into the $O\left(p^{4}\right)$ expression for $\Pi_{6}(s)$, which amounts to replace the lowest order expression for the pseudo-scalar masses there by their expression up to $O\left(p^{4}\right)$. Formula (2.23) in the text is obtained from $B+C$, setting $m=0$. 

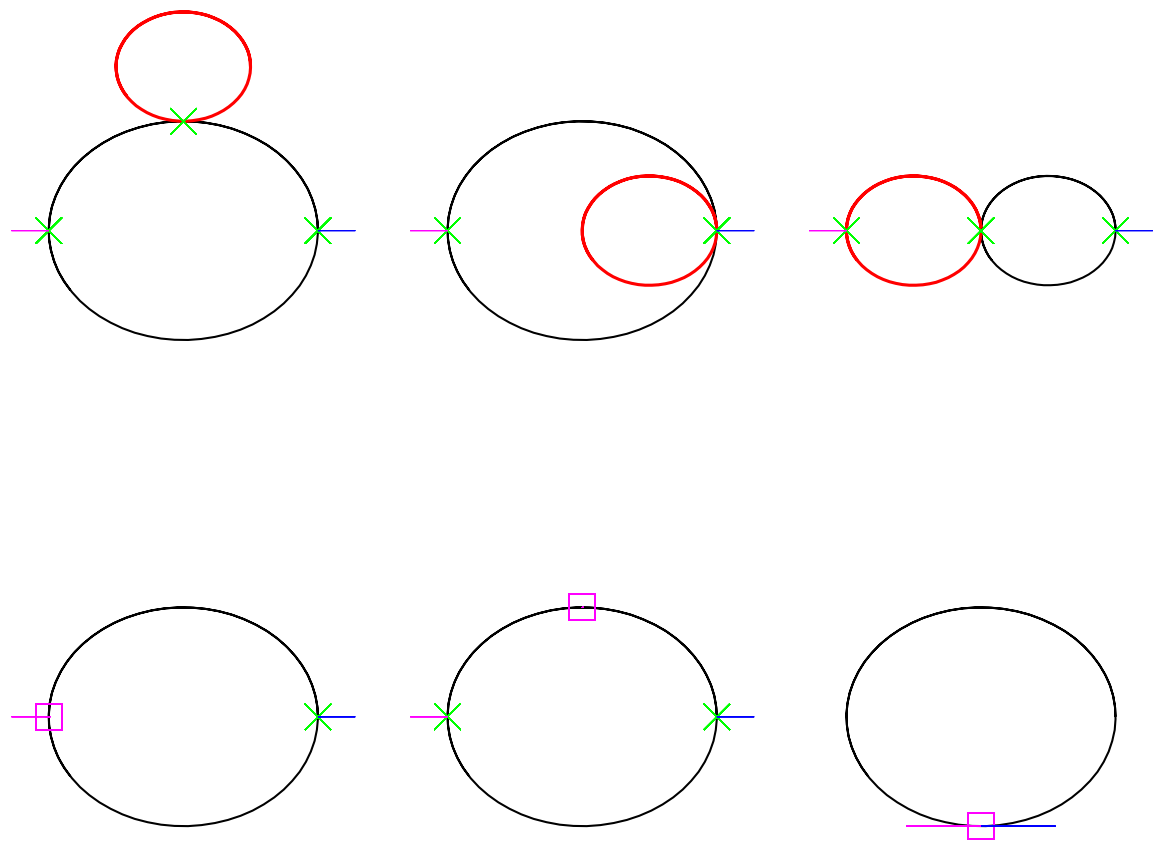

Figure 1: Set of one and two-loops Feynman graphs contributing at chiral order $p^{6}$ to the correlation function $\Pi_{6}(s)$ : crosses denote vertices from the $O\left(p^{2}\right)$ chiral lagrangian, boxes denote vertices from the $O\left(p^{4}\right)$ chiral lagrangian. Some diagrams obtained by exchanging external lines have not been drawn.

\section{References}

[1] J. Gasser and H. Leutwyler, Nucl. Phys. B 250 (1985) 465.

[2] Y. Iwasaki et al., Prog. Theor. Phys. Suppl. 131 (1998) 415.

[3] R.D. Mawhinney, Nucl. Phys. (Proc. Sup.) 60A (1998) 306, C. Sui, Nucl. Phys. (Proc. Sup.) 73 (1999) 228.

[4] G. Ecker, J. Gasser, A. Pich and E. de Rafael, Nucl. Phys. B 321 (1989) 311.

[5] The Particle Data Group, C. Caso et al., Eur. Phys. Jour. C3 (1998) 1.

[6] S. Descotes, L. Girlanda and J. Stern, J. High Energy Phys. 0001 (2000) 041

[7] B. Moussallam, Z. Physik C 14 (2000) 111.

[8] J.F. Donoghue, J. Gasser and H. Leutwyler, Nucl. Phys. B 343 (1990) 341. 
[9] J. Bijnens, G. Colangelo and G. Ecker, Ann. Phys. (NY) 280 (2000) 100.

[10] J. Bijnens, G. Colangelo and G. Ecker, J. High Energy Phys. 9902 (1999) 020.

[11] E. Golowich and J. Kambor, Phys. Rev. D 58 (1998) 036004.

[12] G. Amoros, J. Bijnens and P. Talavera, Nucl. Phys. B 568 (2000) 319.

[13] M. Gell-Mann and M. Lévy, Nuov. Cim. 16 (1960) 705, M. Lévy, Nuov. Cim.52 (1967) 23.

[14] D. Vautherin and T. Matsui, Phys. Rev. D 55 (1997) 4492, Y. Tsue, D. Vautherin and T. Matsui, Prog. Theor. Phys. 102 (1999) 313.

[15] J. Schaffner-Bielich and J. Randrup, Phys. Rev. C 59 (1999) 3329.

[16] A.A. Anselm, Phys. Lett. B 217 (1989) 169, A.A. Anselm and M.G. Ryskin, Phys. Lett. B 266 (1991) 482, J.D. Bjorken, Int. Jour. Mod. Phys. A7 (1992) 4819, Acta. Phys. Pol. B23 (1992) 501, J.P. Blaizot and A. Krzywicki, Phys. Rev. D 46 (1992) 246,

K. Rajagopal and F. Wilczek, Nucl. Phys. B 404 (1993) 577.

[17] K. Symanzik, Comm. Math. Phys. 16 (1970) 48.

[18] J.L. Basdevant, C.D. Froggatt and J.L. Petersen, Phys. Lett. B 41 (1972) 178.

[19] E. van Beveren et al., Z. Physik C 30 (1986) 615.

[20] D. Black, A.H. Fariborz, F. Sannino and J. Schechter, Phys. Rev. D 58 (1998) 054012.

[21] K.L. Au, D. Morgan and M.R. Pennington, Phys. Rev. D 35 (1987) 1633.

[22] R. Kamiński, L. Leśniak and B. Loiseau, Phys. Lett. B 413 (1997) 130.

[23] J.F. Donoghue and E. Golowich, Phys. Rev. D 49 (1994) 1513.

[24] P. Minkowski and W. Ochs, Eur. Phys. Jour. C9 (1999) 283.

[25] J. Stern, hep-ph/9801282.

[26] I.I. Kogan, A. Kovner and M. Shifman, Phys. Rev. D 59 (1999) 016001.

[27] S. Coleman, J. Wess and B. Zumino, Phys. Rev. 177 (1969) 2239, C. Callan, S. Coleman, J. Wess and B. Zumino, Phys. Rev. 177 (1969) 2247.

[28] J. Gasser and H. Leutwyler, Ann. Phys. (NY) 158 (1984) 142.

[29] M. Ishida, Prog. Theor. Phys. 101 (1999) 661.

[30] M. Napsuciale, hep-ph/9803396.

[31] N.A. Törnqvist, Eur. Phys. Jour. C11 (1999) 359.

[32] S. Weinberg, Phys. Rev. Lett. 18 (1967) 188. 
[33] D. Black, A.H. Fariborz, F. Sannino and J. Schechter, Phys. Rev. D 59 (1999) 074026.

[34] L.H. Chan and R.W. Haymaker, Phys. Rev. D 10 (1974) 4143.

[35] M. Pennington, Talk given at "Workshop on Hadron Spectroscopy (WHS 99)", Rome (1999), hep-ph/9905241.

[36] G. Amoros, J. Bijnens and P. Talavera, Phys. Lett. B 480 (2000) 71.

[37] G. Amoros, J. Bijnens and P. Talavera, hep-ph/0003258.

[38] T. Schäfer and E.V. Shuryak, Phys. Rev. D 53 (1995) 6522. 